\title{
Municipal Waste: Effect on the Physico-Chemical and Microbiological Parameters of Otamiri River in Owerri, Nigeria
}

Eze Chinwe Catherine ${ }^{1 *}$, Ekekwe Evelyn Nkechi ${ }^{2}$, Ezenwata Ifeoma Susan ${ }^{3}$, Frank$^{-}$Ogu Ngozi ${ }^{1}$, Okorie I. Ugochukwu $^{1}$, Anaebonam Emeka ${ }^{3}$, Nweze Kenneth Emeka ${ }^{3}$ \& Onyemeka Regland Michael ${ }^{4}$ ${ }^{I}$ Department of Microbiology, Federal University of Technology, Owerri, Nigeria. ${ }^{2}$ Department of Medical Laboratory Sciences, Nnamdi Azikiwe
University-Nnewi, Anambra, Nigeria. ${ }^{3}$ Department of Biological Sciences, Chukwuemeka Odumegwu Ojukwu University, Anambra, Nigeria. ${ }^{4}$ Department of Botany, Lagos State University, Lagos, Nigeria. Email: eze2chinwe@yahoo.com*

DOI: http://doi.org/10.38177/AJBSR.2021.3310

Copyright: $\odot 2021$ Eze Chinwe Catherine et al. This is an open access article distributed under the terms of the Creative Commons Attribution License, which permits unrestricted use, distribution, and reproduction in any medium, provided the original author and source are credited.

Water pollution has deleterious effects as hazards to human health, living resources and hindrances to marine activities. Therefore this research aimed to ascertain the influence of municipal solid waste on physicochemical parameters and microbiological parameters of water samples obtained from Otamiri River. Stream samples were collected at five different points. The physicochemical and microbiological parameters of samples collected were analyzed. Most of the physicochemical parameters were high for some of the locations. The bacteriological analysis result shows a high bacteria count in the water which can be attributed to leachate from dumped human faeces in the river banks. At $P<0.05$, acidity correlated negatively with THBC $(r=-0.903)$, TCC $(r=-0.449), F C C(r=-0.594)$. TSS correlated positively with E.coli $(r=0.938)$. PO4 3 correlated positively with THBC ( $r=0.905)$. At $P<0.01$, Turbidity correlated positively with THBC ( $r=0.997)$. Dissolved Oxygen correlated negatively with all the physicochemical parameters. BOD correlated negatively with TCC ( $r=-0.877)$, E.coli $(r=-0.929), F C C(r=-0.742)$, TFC $(r=-0.117)$. Total hardness correlated positively with FCC $(r=0.975)$, PO4 3 correlated positively with E. coli $(r=0.989)$ and $\mathrm{FCC}_{(}(r=0.967)$. NO 3 correlated positively with E. coli $(r=0.976)$. Summarily, the proximity of the waste dump to Otamiri River has certainly shown considerable physical, chemical, and most importantly, biological effects on its water quality. The presence of the organism shows that the river requires auxiliary treatment before usage as a source of drinking water.

Keywords: Physico-chemical, Microbiological, Waste disposal, Water pollution.

\section{Introduction}

Water is necessary to live on earth as all organisms contain it, some live in it and others drink it. Plants and animals require moderately pure water, and they cannot survive if their water is loaded with toxic chemicals or harmful microorganisms. If severe, water pollution can kill large numbers of fish, birds, and in some cases, killing all members of a species in an affected area. The level of its physical, chemical and biological impurities defines the quality of the water. Water quality is evaluated relative to its intended use. Extensive scrutiny of water used for diverse activities is necessary because numerous pathogens can live in them especially waters used as a source of drinking water like streams, lakes, ponds, and oceans [1].

The major water pollutants are chemical, biological, or physical materials that degrade water quality. Most developing countries are facing new constraints and pressure about solid waste management [2]. One common method of water disposal in Nigeria, which harms the surface water, is illegal (open) dumping. Population explosion, uncontrolled urbanization, and industrialization have caused a high rate of waste generation in Nigeria [3]; even as Ezeronye and Ubalua, [4] pointed out that aquatic pollution problems in Nigeria are on the increase.

Owerri is drained by two rivers, Nworie River and Otamiri Rivers, the later stretching its length to Etche in Rivers State from where it flows into the Atlantic Ocean. Industrial and commercial activities such as sand and gravel mining, landfills, a motor mechanic village among others are all located along the Otamiri watershed. Of paramount importance is the setting of refuse dump along the watercourse, Fakayode [5]. The initial effect of waste 
is to degrade the physical quality of the water. Later, biological degradation becomes evident in terms of the number, variety, and organization of the living organisms in the water.

The nature of pathogens and their effects on humans is advanced - and continually advancing [6]. Aside from human health concerns, the input of waste into water bodies may not always hurt the aquatic environment because of the self-purification property of the water bodies but the indiscriminate dumping of wastes into natural water bodies can overtax this self-purifying capacity of the receiving water [7]. This will not only endanger the residents aquatic lives but may also impair other amenity purposes and non-consumption uses [8].

Nworie River, a first-order stream runs about a $5 \mathrm{~km}$ course across Owerri metropolis in Imo State, Nigeria before emptying into the Otamiri River. The Otamiri watershed is subject to intensive human and industrial activities resulting in the discharge of a wide range of pollutants. When the public water supply fails, the river further serves as a source of direct drinking water, especially for the poorer segment of the city. Microbial contamination of drinking water has a severe health impact on infants and young children in many developing regions of the world, where childhood diarrheal diseases from ingesting polluted water are the second most common cause of infant death. According to Cookey et al. [9], people who ingest polluted water can become ill, and, with prolonged exposure, may develop cancers or bear children with birth defects. The resultant effects of this on public health and the environment are usually great in magnitude.

However, the occurrence of these environmental problems depends on the presence of certain pathogenic microorganisms, which could be contributed by such anthropogenic establishments as municipal solid waste dumpsites, similar to the one located proximally to the Otamiri River in the Owerri Municipal council. This research is therefore an attempt to determine possible contributions of pathogenic microorganisms and other physiochemical imbalances in the Otamiri River by a solid waste dumpsite in its neighborhood. More specifically, this research aimed to ascertain the influence of municipal solid waste on physicochemical parameters and microbiological parameters of water samples obtained from Otamiri River.

\section{Materials and Method}

\section{Area of Study}

Owerri, the study area is the capital city of the Imo State, Nigeria. Owerri lies within latitude $05^{\circ} 29^{\prime} 06 \mathrm{~s}$ and longitude $07^{\circ} 02^{\prime} 06 \mathrm{~s}$ (Fig. 1). The area experiences a longer wet (which lasts from April to November) than the dry season (which lasts the rest of the year) (Victor et al. 2011). The Otamiri River is the major river that courses through the Ihiagwa Community in the Owerri West Local Government Area of Imo State, Nigeria. It runs from Egbu where it has its major base, through to Nekede, Ihiagwa, Eziobodo, Olakwu, Umuisi, Mgbirichi, Umuagwo and finally to Ozuzu in Etche town of Rivers State where it finally joins the Atlantic Ocean [3].

\section{Research Design}

A semi-experimental field research design was used for the study. A semi-experimental field research design allows a researcher to collect original data in a natural setting allowing experimentation without introducing confounding variables. The independent variable includes physicochemical. 


\section{Sample collection}

Water samples from Otamiri River were obtained from locations (five communities) closest to landfill, mechanical workshop village and sand and gravel mine at the early hours of the day for four months: September, October, November, and December. Samples were taken once every month under aseptic conditions. Site location1 was located 300m from the source of the stream at Egbu village near Owerri and served as the control. Site Location 2 was located at 300m from SL1, and about $31 \mathrm{~m}$ from the dumpsite, while Site Location 3 was located downstream behind Emmanuel College premises, at about 300m from Site Location 2. Finally, the sample collected at Site Location 4 was a composite sample collected from the riverbanks around Nekede village; about $310 \mathrm{~m}$ from Sample Location 3 and at $1 \mathrm{~m}$ below the water surface. Sample Location 1 and Sample Location 5 represent the downstream and upstream samples respectively. Sample Location 2, 3, 4 were used to assess the contribution of municipal solid waste and other human activities from the municipality into the Otamiri River. Observations were equally made on the water current and human activities going on along the vicinity of the riverbanks.

\section{Instrument for Sample Collection}

The containers were unscrewed at a depth of $15-30 \mathrm{cmbelow}$ the surface of the water, facing the upstream direction and corked when filled while still underwater to prevent oxidation. A small air space of 2 to $3 \mathrm{~cm}$ above the sample was left in the container for proper mixing of the sample before analysis except for the water samples for biochemical oxidation demand (BOD) and dissolved oxygen (DO) determination. It was tightly closed and labelled. Bottles were rinsed with the river water before sampling. These were stored in the icebox to retard the biochemical activities and promptly transported to the laboratory. The media used in this study were Nutrient agar (Fluka Biochemica, Germany), MacConkey agar (Antec), Sabourand dextrose agar (Fluka Biochemica, Germany), Eosin Methylene Blue (EMB), MF-Endo agar medium, KF- streptococcus agar thiosulphate and citrate bile sucrose agar (TCBS). Nutrient agar medium was used for isolation of aerobic heterotrophic bacteria while total coliform isolates were obtained using MF-Endo agar medium. KF- streptococcus agar was used for faecal streptococci while thiosulphate citrate bile sucrose agar (TCBS) was used to isolate Vibrio species. The enumeration of Salmonella species was carried out using the bismuth sulphate agar method [10]. Media preparations were according to manufacturer's instructions and incubation generally, except for faecal coliform, was at $35-37^{\circ} \mathrm{C}$ for $24 \mathrm{~h}$. All glassware and media used were sterilized by autoclaving at $121^{\circ} \mathrm{C}$ for $15 \mathrm{~min}, 15$ pounds pressure and air-drying in the hot air oven at $160^{\circ} \mathrm{C}$ (two hrs.). Of the samples collected, one was immediately analysed for the following parameters in the field: $\mathrm{pH}$, Temperature, Electrical Conductivity Total Dissolved Solids (TDS) and Dissolved Oxygen) using Digital Meters. Serial dilution of the water samples was carried out using sterile distilled water. The samples were analysed as per standard methods mentioned in APHA [11]. All the different dilutions were properly labelled and used for total plate count. The standards reagents used in the analysis were prepared using double distilled water.

\section{Sample Analyses of Physico-Chemical Parameters}

The BOD was evaluated by first diluting the sample and incubating it in the dark at $20^{\circ} \mathrm{C}$ for five days and measuring the amount of oxygen consumed. The DO sample was fixed with $2 \mathrm{ml}$ each of Wrinkler I and II reagents 
at the site, while $\mathrm{BOD}_{5}$ sample bottles were corked and wrapped with aluminum foil and the $\mathrm{BOD}_{5}$ sample was fixed with the Wrinkler method after five days of incubation at room temperature $\left(25^{\circ} \mathrm{C}\right)$ in the dark place. Conductivities were measured at $25^{\circ} \mathrm{C}$ directly in $\mu \mathrm{s} / \mathrm{cm}$ using a digital conductivity meter (NorLab; LM8). Turbidities were measured with a digital turbidimeter (Hach Chemicals; 2100P). The total suspended and total dissolved solids were separated by filtering the water through a $0.45 \mu \mathrm{m}$ filter paper and determined according to standard procedures [12]. Acidity and alkalinity were determined by titration methods. Dissolved oxygen was determined using a dissolved oxygen meter; In this case, the dissolved oxygen (DO) of the samples was determined using the Schott Gerate Dissolved Oxygen meter and then incubated for five (5) days at 200C. DO was again measured after five days and BOD in $\mathrm{mg} / 1$ was determined from the following calculation and reported accordingly. $\mathrm{BOD}_{5}$ was determined using $\mathrm{BOD}_{5}$ Track; electrical conductivity was measured by electrical conductivity meter - DIST3 by HANNA limited. Ammonia - Nitrogen $\left(\mathrm{NH}_{3}-\mathrm{N}\right)$ was determined by direct Nesslerization for natural effluent and Nitrate - Nitrogen $\left(\mathrm{NO}_{3}-\mathrm{N}\right)$ was determined by the Brucine method [13]. Their absorbance values were read with UNICAM 8626 UV/VIS spectrophotometer at $470 \mathrm{~nm}$ for $\mathrm{NO}_{3}-\mathrm{N}_{\text {and }} \mathrm{PO}_{4}$ and $420 \mathrm{~nm}$ for $\mathrm{SO}_{3}$ ions.

\section{Test for the presence of nitrite}

The test for the presence of nitrite was carried out using a modification of the method described by [14] which involves Greiss-Ilosvay reagent. Suspensions had to be prepared by dissolving some part of the isolated cultures from the selective medium in $1 \mathrm{ml}$ of sterile deionised water.

\section{Test for Temperature}

This was determined at the point of sample collection by dipping the bulb of mercury-in-glass thermometer into the soil suspension and recording the readings. Reading was taken when there was a steady temperature from the calibration on the capillary tube.

\section{Conductivity Test}

This was measured using the suntex conductivity meter (DD 193). After it was calibrated, the electrode was inserted into the portion of the sample and the conductivity button was pressed. The valve was read.

\section{Test for Phosphorus}

This was determined using Vanado-molybdophosphoric acid colourimetric method using ammonium molybdate which formed molybdo-phoshoric acid under acidic conditions. The intensity of the yellow colour was measured using a spectrophotometer at $490 \mathrm{~nm}$.

\section{Result}

\section{Parameters of Samples}

The Physico-chemical parameters of samples from the predetermined spots in Otamiri River, Imo State, Nigeria are presented in Table 1. After incubation, the colonies were enumerated macroscopically and the results of the microbial population from soil samples from the five site locations are shown in Table 2. 
Table 1. Descriptive statistics of the physicochemical parameters

\begin{tabular}{|c|c|c|c|c|c|}
\hline Parameters & Minimum & Maximum & Range & Mean & SE \\
\hline $\mathrm{pH}$ & 6.23 & 7.12 & .89 & 6.5240 & .17137 \\
\hline TSS & 112.80 & 568.60 & 455.80 & 320.5400 & 90.42545 \\
\hline EC & 60.13 & 154.30 & 94.17 & 87.6780 & 17.46188 \\
\hline Acidity & 28.45 & 32.44 & 3.99 & 30.0820 & .66453 \\
\hline TDS & 49.00 & 67.00 & 18.00 & 55.4000 & 3.31059 \\
\hline Turbidity & 10.60 & 110.24 & 99.64 & 72.7260 & 17.94098 \\
\hline DO & 3.76 & 5.60 & 1.84 & 4.6760 & .31752 \\
\hline BOD & 2.50 & 3.53 & 1.03 & 2.9200 & .21324 \\
\hline $\mathrm{Cl}$ & 20.20 & 120.60 & 100.40 & 63.6000 & 20.14229 \\
\hline Alkalinity & 30.24 & 37.33 & 7.09 & 32.6960 & 1.30086 \\
\hline T. Hardness & 39.27 & 98.27 & 59.00 & 69.8240 & 11.63673 \\
\hline $\mathrm{Ca}$ & 1.47 & 64.24 & 62.77 & 16.1780 & 12.04413 \\
\hline $\mathrm{Mg}$ & 2.24 & 3.60 & 1.36 & 2.6820 & .27240 \\
\hline K & 2.54 & 2.73 & .19 & 2.5980 & .03455 \\
\hline $\mathrm{Na}$ & 3.27 & 5.27 & 2.00 & 4.5540 & .35413 \\
\hline PO4 & .10 & 10.23 & 10.13 & 5.5120 & 1.96288 \\
\hline $\mathrm{SO} 4$ & 1.98 & 2.66 & .68 & 2.3940 & .12883 \\
\hline NO3 & 2.80 & 4.22 & 1.42 & 3.5840 & .28335 \\
\hline NH3 & .003 & .100 & .097 & .03740 & .018041 \\
\hline Temperature & 25.00 & 29.00 & 4.00 & 27.1400 & .76785 \\
\hline $\mathrm{Fe}$ & .14 & 1.04 & .90 & .4240 & .16080 \\
\hline
\end{tabular}

$\mathrm{SE}=$ standard error of mean, TSS=Total Suspended Solids, EC=Electrical Conductivity, TDS= Total Dissolved Solids, Do=Dissolved Oxygen, BOD $=$ Biological Oxygen Demand, $C L=$ Chlorides, $\mathrm{T}$ hardness $=$ Total Hardness, $\mathrm{Ca}=$ Calcium, $\mathrm{Mg}=$ Magnesium, $\mathrm{K}=$ Pottasium, $\mathrm{Na}=$ Sodium, $\mathrm{PO}_{4}=$ Phosphates, $\mathrm{SO}_{4}=$ sulphates, $\mathrm{NO}_{3}=\mathrm{Nitrates}$ $\mathrm{NH}_{3}=$ Ammonium, $\mathrm{Fe}=$ Iron, $\mathrm{THBC}=$ Total Heterotrophic Bacterial Count, $\mathrm{TCC}=$ Total Coliform Count, TFC= Total Fecal Count, $\mathrm{COD}=$ Chemical Oxygen Demand. 
Asian Journal of Basic Science \& Research Volume 3, Issue 3, Pages 98-109, July-September 2021

Table 2. Descriptive statistics of the microbial groups of Otamiri River

\begin{tabular}{|lllll|}
\hline Microbial groups & Minimum & Maximum & Mean & SEx10 $^{4}$ \\
\hline THBC $(\mathrm{cfu} / \mathrm{ml})$ & $3.24 \times 10^{5}$ & $6.7 \times 10^{5}$ & $5.4 \times 10^{6}$ & $6.1 \times 10^{4}$ \\
\hline TCC $(\mathrm{cfu} / \mathrm{ml})$ & $2.5 \times 10^{4}$ & $3.74 \times 10^{4}$ & $3.30 \times 10^{4}$ & $2.20 \times 10^{3}$ \\
\hline E.coli $(\mathrm{MPN} / 100 \mathrm{ml})$ & 1.39 & 3.80 & 2.68 & 0.52 \\
\hline FCC $(\mathrm{cfu} / \mathrm{ml})$ & $2.10 \times 10^{2}$ & $3.80 \times 10^{2}$ & $2.92 \times 10^{2}$ & $3.2 \times 10^{1}$ \\
\hline TFC $(\mathrm{cfu} / \mathrm{ml})$ & $2.49 \times 10^{3}$ & $3.4 \times 10^{3}$ & $3.02 \times 10^{3}$ & $1.79 \times 10^{2}$ \\
\hline
\end{tabular}

$\mathrm{SE}=$ standard error of mean, $\mathrm{THBC}=$ Total Heterotrophic Bacterial Count, $\mathrm{TCC}=$ Total Coliform Count, $\mathrm{FCC}=$ Feacal Coliform Count, TFC $=$ Total Faecal Count

Table 3 shows microscopic morphology and cultural characteristics of fungal isolates from soil samples. Aspergillus spp, Fusarium spp, Penicillin spp, and Mucor, Candida spp, Rhizopus spp, and Norcadia spp were the fungal isolates identified.

Table 4 shows biochemical tests of bacteria isolates from the soil samples. The bacterial genera isolated were Pseudomonas, Serratia, Shigella, Bacillus, Staphylococcus, Escherichia, Proteus, Klebsiella, Streptococcus, Micrococcus, and Corynebacterium spp.

Table 3. Microscopic morphology, cultural characteristics and \% occurrence of fungal isolates from Otamiri River

\begin{tabular}{|c|c|c|c|c|c|c|c|}
\hline \multirow[t]{2}{*}{ Organism } & \multirow[t]{2}{*}{ Microscopic morphology } & \multirow{2}{*}{$\begin{array}{l}\text { Cultural } \\
\text { characteristics }\end{array}$} & \multicolumn{5}{|c|}{ Occurrence (\%) } \\
\hline & & & SL1 & SL2 & SL3 & SL4 & SL5 \\
\hline $\begin{array}{l}\text { Aspergillus } \\
\text { niger }\end{array}$ & $\begin{array}{l}\text { Has septate hyphae with long and } \\
\text { smooth conidiophores, large, round } \\
\text { unbranched sporangiophores }\end{array}$ & $\begin{array}{l}\text { Golden reverse side, } \\
\text { creamy and brownish } \\
\text { mycelium, powdery }\end{array}$ & 20 & 21 & 17 & 24 & 18 \\
\hline $\begin{array}{l}\text { Aspergillus } \\
\text { flavus }\end{array}$ & $\begin{array}{l}\text { Colourless, long, erect swollen } \\
\text { conidiophores and septate hyphae }\end{array}$ & $\begin{array}{l}\text { Colonies are Greenish } \\
\text { yellow colour with } \\
\text { creamy edge }\end{array}$ & 19 & 18 & 24 & 19 & 21 \\
\hline $\begin{array}{l}\text { Fusarium } \\
\text { species }\end{array}$ & $\begin{array}{l}\text { Dark pigmented conidiophores, } \\
\text { spherical }\end{array}$ & $\begin{array}{l}\text { Powderish and creamy } \\
\text { colonies }\end{array}$ & 20 & 17 & 22 & 23 & 18 \\
\hline $\begin{array}{l}\text { Penicillin } \\
\text { species }\end{array}$ & $\begin{array}{l}\text { Fruity mycelium, branched } \\
\text { conidiophores with white margin }\end{array}$ & $\begin{array}{l}\text { Greenish and } \\
\text { filamentous colonies }\end{array}$ & 19 & 18 & 17 & 26 & 20 \\
\hline $\begin{array}{l}\text { Mucor } \\
\text { pusillus }\end{array}$ & $\begin{array}{l}\text { Non septate hyphae with short } \\
\text { sporangiophores }\end{array}$ & $\begin{array}{l}\text { Creamy and irregular } \\
\text { colonies }\end{array}$ & 22 & 18 & 23 & 25 & 12 \\
\hline
\end{tabular}


Table 4. Biochemical tests and percentage occurrence of bacteria isolates from Otamiri River

\begin{tabular}{|c|c|c|c|c|c|c|c|c|c|c|c|c|c|c|c|c|}
\hline \multirow{2}{*}{ Organism } & \multirow{2}{*}{$\begin{array}{c}\text { Gram } \\
\text { Reaction }\end{array}$} & \multirow{2}{*}{ Cultural Characteristics } & \multirow{2}{*}{ 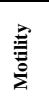 } & \multirow{2}{*}{ 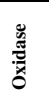 } & \multirow{2}{*}{ 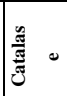 } & \multirow{2}{*}{ } & \multirow{2}{*}{ 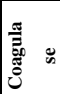 } & \multirow{2}{*}{ 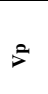 } & \multirow{2}{*}{$\dot{\Sigma}$} & \multirow{2}{*}{ 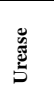 } & \multirow{2}{*}{$\frac{\mathscr{2}}{\square}$} & \multicolumn{5}{|c|}{ Occurrence (\%) } \\
\hline & & & & & & & & & & & & SL1 & SL2 & SL3 & SL4 & SL5 \\
\hline Pseudomonas sp & $\begin{array}{l}\text { Negative } \\
\text { rods }\end{array}$ & Blue green colonies on $\mathrm{NA}$ & + & + & + & + & - & - & + & - & - & 8 & 10 & 28 & 35 & 19 \\
\hline Shigella & $\begin{array}{l}\text { negative } \\
\text { rods }\end{array}$ & Pink colonies on DCA & + & - & - & - & - & - & + & - & - & 10 & 15 & 24 & 28 & 23 \\
\hline Serratia sp & $\begin{array}{l}\text { Negative } \\
\text { rod }\end{array}$ & $\begin{array}{l}\text { Red, circular entire margin } \\
\text { colonies }\end{array}$ & + & + & + & + & - & + & - & - & - & 10 & ns & ns & 43 & 47 \\
\hline Bacillus sp & $\begin{array}{l}\text { Positive } \\
\text { rod }\end{array}$ & Milky white colonies & + & + & + & + & - & + & - & $-1+$ & - & 15 & 17 & 38 & 20 & 10 \\
\hline Staphylococcus sp & $\begin{array}{l}\text { Positive } \\
\text { cocci }\end{array}$ & $\begin{array}{l}\text { Round, golden-yellow colonies, } \\
\text { often with hemolysis, on blood } \\
\text { agar plates }\end{array}$ & - & - & + & - & + & + & - & - & - & 14 & 15 & 25 & 30 & 16 \\
\hline Corynebacterium sp & $\begin{array}{l}\text { Positive } \\
\text { rod }\end{array}$ & & - & - & + & + & - & & & - & + & 12 & 15 & 25 & 26 & 22 \\
\hline E.coli & $\begin{array}{l}\text { Negative } \\
\text { rods }\end{array}$ & Small white colonies & + & + & + & - & - & - & + & - & + & 5 & 11 & 28 & 23 & 17 \\
\hline Proteus Spp & $\begin{array}{l}\text { Positive } \\
\text { rods }\end{array}$ & Large milky white & + & - & + & + & - & + & - & + & - & 8 & 11 & 31 & 27 & 23 \\
\hline Kleb Spp & -rods & $\begin{array}{l}\text { Slightly gummy colonies, } \\
\text { circular, convex, entire margin }\end{array}$ & + & - & - & + & - & + & - & + & - & 10 & 15 & 25 & 22 & 28 \\
\hline Streptococcus Sp & + cocci & & - & - & - & - & & & & - & - & 5 & 10 & 30 & 33 & 22 \\
\hline Micrococcus Sp & $\begin{array}{l}\text { Positive } \\
\text { cocci }\end{array}$ & Yellow punctiform colonies & - & & + & & & & & & & 3 & $\mathrm{~ns}$ & 36 & 39 & 22 \\
\hline
\end{tabular}

(+ =positive; - negative reactions $)$ ns= not seen

Table 5. Correlations (r) matrix (MPN/100ml) between the physicochemical parameter and microbial groups

\begin{tabular}{|c|c|c|c|c|c|c|c|c|c|c|c|c|c|c|c|c|c|c|c|c|c|}
\hline$\sum_{\exists}^{\bar{J}}$ & II & $\omega_{1}^{2}$ & نI & $\begin{array}{l}0 \\
\frac{0}{2} \\
\end{array}$ & $\hat{\hat{\theta}}$ & 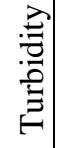 & ○ & Оి & $\frac{1}{U}$ & 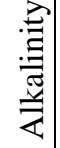 & 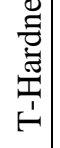 & $\tilde{U}$ & $\sum^{\infty}$ & 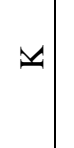 & $\tilde{z}$ & $\begin{array}{l}\tilde{m} \\
\dot{2}\end{array}$ & $\begin{array}{c}\text { Tे } \\
\text { O }\end{array}$ & $\begin{array}{l}0 \\
z\end{array}$ & $\stackrel{\mathbf{Z}}{\mathbf{Z}}$ & 害 & $\stackrel{0}{ \pm}$ \\
\hline $\begin{array}{l}\text { U } \\
\stackrel{M}{1}\end{array}$ & $\begin{array}{l}\text { oo } \\
\stackrel{0}{0}\end{array}$ & $\stackrel{\mathfrak{n}}{\stackrel{0}{0}}$ & $\begin{array}{l}\tilde{n} \\
\stackrel{0}{0}\end{array}$ & $\begin{array}{l}\hat{\delta} \\
\hat{o} \\
\hat{1}\end{array}$ & 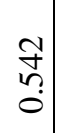 & $\begin{array}{l}\text { *. } \\
\stackrel{+}{\hat{\alpha}} \\
\grave{0}\end{array}$ & $\begin{array}{l}2 \\
\stackrel{2}{0} \\
\dot{1}\end{array}$ & $\frac{\mathfrak{r}}{\mathfrak{s}}$ & $\begin{array}{l}\begin{array}{l}+ \\
0 \\
0 \\
0 \\
0\end{array}\end{array}$ & $\begin{array}{l}9 \\
\mathfrak{1} \\
\vdots \\
0\end{array}$ & $\begin{array}{l}\tilde{b} \\
\hat{0}\end{array}$ & $\begin{array}{l}0 \\
\infty \\
? \\
0\end{array}$ & 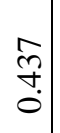 & 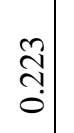 & $\begin{array}{c}f \\
⿱ 乛 \\
\infty \\
0\end{array}$ & 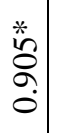 & $\begin{array}{l}* \\
\infty \\
\vdots \\
\vdots\end{array}$ & $\begin{array}{l}3 \\
0 \\
0 \\
0\end{array}$ & \begin{tabular}{l}
0 \\
\multirow{1}{*}{} \\
$\vdots$ \\
0
\end{tabular} & 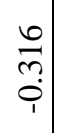 & $\begin{array}{l}a \\
a \\
o\end{array}$ \\
\hline$\underset{U}{U}$ & $\begin{array}{l}\tilde{n} \\
\tilde{o}\end{array}$ & $\begin{array}{l}\stackrel{0}{0} \\
0 \\
0\end{array}$ & $\begin{array}{l}\stackrel{?}{f} \\
\stackrel{+}{0}\end{array}$ & $\begin{array}{l}0 \\
+ \\
\dot{p} \\
\dot{1}\end{array}$ & 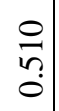 & $\begin{array}{l}\hat{b} \\
n \\
0\end{array}$ & \begin{tabular}{l} 
\pm \\
\multirow{n}{n}{} \\
0 \\
1
\end{tabular} & $\begin{array}{l}\hat{2} \\
0 \\
0 \\
0\end{array}$ & 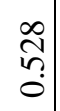 & $\begin{array}{c}\frac{d}{f} \\
\dot{0}\end{array}$ & 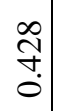 & \begin{tabular}{l}
$\tilde{\jmath}$ \\
\multirow{\leftarrow}{0}{}
\end{tabular} & $\begin{array}{c}\mathcal{Y} \\
\text { ?̦ }\end{array}$ & $\stackrel{\vec{m}}{0}$ & $\begin{array}{l}\tilde{n} \\
0 \\
0\end{array}$ & $\begin{array}{c}a \\
\dot{0} \\
0 \\
0\end{array}$ & $\begin{array}{l}\hat{a} \\
\tilde{o}\end{array}$ & $\begin{array}{l}\bar{b} \\
\dot{0} \\
0\end{array}$ & $\begin{array}{l}0 \\
⿱ 亠 \\
\dot{0} \\
0\end{array}$ & $\begin{array}{l}\infty \\
2 \\
? \\
0\end{array}$ & $\begin{array}{l}a \\
\stackrel{0}{0} \\
\stackrel{0}{0}\end{array}$ \\
\hline 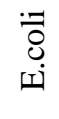 & 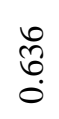 & $\stackrel{\infty}{\infty}$ & 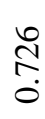 & $\begin{array}{l}\stackrel{0}{\mathfrak{0}} \\
\dot{0}\end{array}$ & $\begin{array}{l}\stackrel{0}{0} \\
\vdots \\
0\end{array}$ & $\begin{array}{l}0 \\
\vdots \\
0\end{array}$ & $\begin{array}{l}\bar{n} \\
0 \\
0 \\
0 \\
1\end{array}$ & $\begin{array}{l}\hat{\grave{\hat{V}}} \\
\hat{i}\end{array}$ & $\begin{array}{l}\hat{\hat{D}} \\
0 \\
0\end{array}$ & 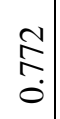 & $\begin{array}{l}\infty \\
0 \\
\infty \\
0 \\
0\end{array}$ & $\begin{array}{l}n \\
\tilde{n} \\
0\end{array}$ & 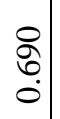 & 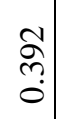 & $\begin{array}{l}n \\
\stackrel{n}{r} \\
\tilde{o}\end{array}$ & 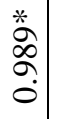 & $\begin{array}{l}\stackrel{*}{o} \\
\dot{\sigma} \\
\dot{0}\end{array}$ & $\begin{array}{l}* \\
0 \\
\vdots \\
\vdots \\
0\end{array}$ & $\begin{array}{l}\infty \\
0 \\
0 \\
0\end{array}$ & $\begin{array}{l}\tilde{b} \\
\dot{0}\end{array}$ & $\begin{array}{l}0 \\
\stackrel{2}{1} \\
0 \\
1\end{array}$ \\
\hline$\underset{I}{U}$ & $\stackrel{\vec{\infty}}{\stackrel{0}{0}}$ & $\frac{ \pm}{\sigma}$ & $\begin{array}{l}\hat{\sigma} \\
\infty \\
0 \\
0\end{array}$ & $\begin{array}{l}+ \\
\stackrel{5}{n} \\
i\end{array}$ & $\begin{array}{l}\hat{\sigma} \\
\dot{0} \\
0\end{array}$ & 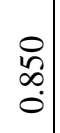 & 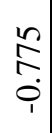 & 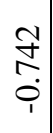 & \begin{tabular}{l}
0 \\
\multirow{2}{0}{} \\
$\dot{0}$
\end{tabular} & 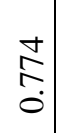 & $\begin{array}{l}\frac{*}{*} \\
\hat{w} \\
\hat{a} \\
\vdots \\
0\end{array}$ & $\begin{array}{c}\tilde{\imath} \\
\stackrel{0}{0}\end{array}$ & $\begin{array}{l}T \\
\infty \\
\dot{0}\end{array}$ & $\begin{array}{l}\text { } \\
\text { ?n } \\
0\end{array}$ & $\begin{array}{c}f \\
⿱ \\
\infty \\
0 \\
\dot{0}\end{array}$ & 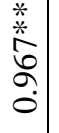 & $\begin{array}{l}* \\
\infty \\
\infty \\
\infty \\
0 \\
0\end{array}$ & $\begin{array}{l}\mathfrak{f} \\
\stackrel{0}{0}\end{array}$ & $\begin{array}{c}m \\
\infty \\
0 \\
0\end{array}$ & $\begin{array}{l}\infty \\
\infty \\
\infty \\
0\end{array}$ & $\begin{array}{l}n \\
0 \\
0 \\
0 \\
i\end{array}$ \\
\hline U & $\begin{array}{l}\hat{\sigma} \\
0 \\
0\end{array}$ & $\widetilde{N}$ & 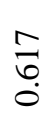 & 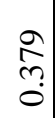 & $\begin{array}{l}8 \\
\\
0\end{array}$ & $\begin{array}{l}0 \\
0 \\
0 \\
1\end{array}$ & $\begin{array}{l}0 \\
0 \\
0 \\
0 \\
1\end{array}$ & $\begin{array}{l}a \\
\vdots \\
0 \\
1\end{array}$ & 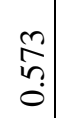 & $\begin{array}{c}\hat{\jmath} \\
\stackrel{5}{0}\end{array}$ & $\begin{array}{l}0 \\
\text { ñ. } \\
0\end{array}$ & $\begin{array}{l}\bar{n} \\
\tilde{n}\end{array}$ & $\begin{array}{l}\stackrel{\partial}{\hat{\sigma}} \\
\dot{0}\end{array}$ & $\begin{array}{l}n \\
= \\
0\end{array}$ & $\begin{array}{l}t+ \\
\stackrel{t}{0}\end{array}$ & $\frac{\Xi}{3}$ & $\begin{array}{l}m \\
\overrightarrow{0}\end{array}$ & $\begin{array}{l}\bar{n} \\
\tilde{o}\end{array}$ & $\begin{array}{c}m \\
\mathfrak{n} \\
0\end{array}$ & 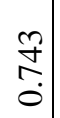 & $\begin{array}{l}0 \\
\delta \\
0 \\
0\end{array}$ \\
\hline
\end{tabular}

$*=$ significant at $\mathrm{p}<0.05, * *=$ significant at $\mathrm{p}<0.01$. 
The Pearson correlations between the physiochemical parameters and the microbial groups are shown in Table 5. At $\mathrm{P}<0.05$, acidity correlated negatively with THBC $(\mathrm{r}=-0.903)$, TCC $(\mathrm{r}=-0.449)$, FCC $(\mathrm{r}=-0.594)$. TSS correlated positively with E.coli $(\mathrm{r}=0.938)$. $\mathrm{PO}_{3}$ correlated positively with $\mathrm{THBC}(\mathrm{r}=0.905)$. At $\mathrm{P}<0.01$, Turbidity correlated positively with THBC $(r=0.997)$. Dissolved Oxygen correlated negatively with all the physiochemical parameters. BOD correlated negatively with TCC $(\mathrm{r}=-0.877)$, E.coli $(\mathrm{r}=-0.929)$, FCC $(\mathrm{r}=$ -0.742), TFC ( $\mathrm{r}=-0.117)$. Total hardness correlated positively with FCC $(\mathrm{r}=0.975), \mathrm{PO}_{3}$ correlated positively with E. coli $(r=0.989)$ and FCC $(r=0.967)$. $\mathrm{NO}_{3}$ correlated positively with E. coli $(r=0.976)$. The test of homogeneity in mean variance of levels of the physicochemical parameters across the sampling locations revealed significant difference.

\section{Discussion}

\section{Physiochemical analysis}

From the analysis of available data, the major findings of this research are as follows: Variations in the $\mathrm{pH}$, colour, turbidity, temperature, hardness and conductivity of the Otamiri River are shown in Table 1.

Turbidity of the water increased greatly from 10.6 NTU at SL1 to 110 NTU at SL4 showing an increase in the concentration of suspended matters in the water sample and soil particles transported by runoff to the river but highest at the point SL4 after input of wastes from the municipal waste dump. Reduction to 88 NTU at the point of leaving is indicative of self-purification by the river.

Significant increases in conductivity may be an indicator that polluting discharges have entered the water. The result of the analysis shows that the $\mathrm{pH}$ varied between $\mathrm{pH} 6.27$ and 7.12, the highest $\mathrm{pH}$ was recorded at point SL5 with a mean temperature range of 24.3 to $25.8^{\circ} \mathrm{C}$. The total hardness of the water was recorded to be highest at SL5. Calcium and Magnesium hardness range from $1.47 \mathrm{mg} / \mathrm{l}$ to $6.24 \mathrm{mg} / \mathrm{l}$ and from 2.24 to $3.6 \mathrm{mg} / \mathrm{l}$ respectively.

Nitrate levels across the sampling locations were progressively high, the highest located at SL 5. This may explain the rich growth of water weeds and plankton around the study area. Farming and dumping of animal waste along the river course might be responsible for these high readings. A recent study of the Nworie and Otamiri rivers in Nigeria showed a strong relationship between nitrate concentration and urbanization. As urbanization increased, so did the nitrate concentration of the rivers [15]. Phosphate levels varied along with the sampling locations, the highest recorded at SL4 (10.23mg/l). These findings agree with the findings of [16]. Crowther et al. [16] opine that increased nutrients especially nitrate and phosphates imply increasing plant bloom, a situation which may lead to eutrophication in future.

The mean concentrations of dissolved oxygen (DO) of Otamiri vary from 3.76 to $5.6 \mathrm{mg} / \mathrm{l}$ with a mean value of $4.6 \mathrm{mg} / \mathrm{l}$. Organic wastes and other nutrient inputs from sewage and industrial discharges, septic tanks and agricultural and urban runoff can result in decreased oxygen levels. Nutrient inputs often lead to excessive algal growth. When the algae die, bacteria decompose the organic matter. Bacterial decomposition consumes a great deal of oxygen. Dissolved oxygen is depleted by the decaying process. The lower level of DO noted at site SL3 may be attributed to increased growth of aerobic bacteria in the presence of large organic matter, due to anaerobiosis. 
The BOD values from SL 3-5 were higher than those of other locations. The oxygen demand is directly proportional to the amount of oxygen waste materials that are to be broken down [18]. This accounts for the oxygen required in the decomposition of organic matter. The concentration of BOD from the water analysis varies from 3.33-5.54 $\mathrm{mg} \mathrm{L}-1$. The huge waste rich in organic matter and nutrients along the course of the river and probably as a result of the increased microbial activity (respiration) occurring during the degradation of the organic matter may be responsible for the high BOD value in the water.

This trend indicates a significant increase in organic input into the river. In their study, Desai and Tank [19] corroborate that high levels of pollutants in river water systems causes an increase in biological oxygen demand (BOD), chemical oxygen demand (COD), total dissolved solids (TDS), total suspended solids (TSS), and faecal coliform and hence make such water unsuitable for drinking, irrigation and aquatic life.

The temperature ranged between $26-29^{\circ} \mathrm{C}$. The mean temperature values of the water samples are not statistically different from each other $(p<0.05)$. Hence, the temperature of the water from Otamiri River could not be implicated as influencing the observed variations in the bacterial population as well as in other physicochemical parameters. However, the observed range of the temperature allows for an optimum proliferation of most of the bacteria isolated from the water samples.

The high TSS and TDS content values of the water show significant direct relationships to the high bacterial population obtained from the water samples. Total Suspended solids increased from $112.8 \mathrm{mg} / \mathrm{l}$ at SL2 to $568.6 \mathrm{mg} / \mathrm{l}$ at SL5 but recorded $120.3 \mathrm{mg} / \mathrm{l}$ at SL 1.

\section{Microbiological analysis}

The bacteriological analysis result shows a high bacteria count in the water which can be attributed to leachate from dumped human faeces in the river banks. The presence of total coliform in water is an indication of faecal contamination and is responsible for most water-borne diseases such as meningitis, cholera and diarrhoea as well as morbidity and mortality among children [20].

On average about $70 \%$ of the total coliforms are of faecal origin. Faecal indicators (Enterococci or coliphage) are microbes whose presence indicates that the water may be contaminated with human or animal wastes. Results of bacteriological analyses including total heterotrophic count, total coliform and thermotolerant coliform counts revealed a high level of faecal pollution of the river. This range is higher than WHO standards. Some genera could be of soil origin while others are of intestinal and hence faecal origin. Bacteria and other pathogens are the greatest threat to the quality of water for domestic consumption [21]. Bacteria originating from human faeces are a leading cause of child mortality, and water is a common transmission route [20].

Total counts of heterotrophic bacteria were highest at SL3 (close to the municipal solid waste) with fairly steady increases in counts of the microorganisms. The profile showed that bacterial populations increased reaching a peak at SL3. Partially eutrophicated sites seem to have higher isolates of these organisms. Run-offs into this river from a government-owned abattoir located about 100 meters on a slope off the bank of the river and emptying into the river may have greatly contributed to its load of coliforms. This situation is in accordance with other reports of 
Keraita et al. [22] who attributed increased bacterial presence notably $S$. aureus populations to the presence of the refuse dump. Activities such as swimming, washing of vehicles and domestic wares take place daily at SL5, unlike site SL1. WHO [23] reveals that any water intended for drinking should contain faecal and Total coliform count of 0 respectively in any $100 \mathrm{ml}$. It was posited that the total number of heterotrophic bacteria reflects the contamination extent by the easily decomposable organic matters, while the faecal coliform bacteria number gives an idea of the contamination size by faecal substance

Results show that bacterial populations increase downstream with a peak at SL5. Escherichia coli, Klebsiella species, Proteus species, Shigella species and Salmonella species belong to the family Enterobacteriaceae (Kistemann et al., 2002). Their presence in water indicates faecal contamination of the water. For instance, Shigella species have been implicated in bacterial dysentery. Proteus species thrives in urinary tract infection in humans. Staphylococcus epidermitis and Bacillus species are pathogenic and non-pathogenic bacteria respectively. Staphylococcus epidermitis implicated with mucous of man and other warm-blooded animals.

Their presence in water signifies waste material contamination of the water body from humans and other warm-blooded animals whereas; Bacillus species are harmless but are saprophytic in nature indicating the presence of decomposing organic materials in a water body. High counts of bacterial load reflect the level of water pollution as it indicates the amount of organic matter present. A major natural source of pollutants is runoff, which carries debris, sand, silt and nutrients into the river that affects colour and turbidity. These activities include the indiscriminate dumping of refuse into the river, channelling of raw sewage, open defecation, discharge of untreated effluents and dumping of industrial waste into the Otamiri River. Enterobacteriaceae are mesophiles most of which grows optimally at a temperature range of $20^{\circ} \mathrm{C}$ and $32{ }^{\circ} \mathrm{C}$. In E. coli counts were recorded higher counts in order in SL4 (3.8), SL5 (3.5) and SL 3(3.22). These values corresponded with the high level of BOD recorded in all the samples, which indicated the presence of microorganisms in them. The river showed a high bacterial count as is characteristic of water bodies receiving organic pollutants. The presence of E.coli is a definite indication of faecal contamination [23].

\section{Conclusion}

Summarily, the proximity of the waste dump to Otamiri River has certainly shown considerable physical, chemical, and most importantly, biological effects on its water quality. The presence of the organism shows that the river cannot be used directly as a source of drinking water; it ranks among water that requires auxiliary treatment. In addition, some of the organisms encountered in the water are potential pathogens contrary to the WHO (2004) recommendation that drinking water should be free of pathogens.

All these observations pose serious public health hazards, as they constitute potential sources of food-borne gastroenteritis, diarrhoea, salmonellosis, dysentery etc. Increases in nutrient levels, river flow, and aeration enhance aerobic decomposition of organic matter and therefore bacterial counts, leading to the deterioration of the aesthetic value of the river. The presence of some of the organisms suggests that materials were being added to the water from other sources likely to be the refuse dumps. The dumps are exposed and hence can be washed into the river during rains or material be carried from it to the water during heavy winds. Based on the major findings in this 
research, it has become imperative to put up some recommendations that are necessary to improve the environmental and health conditions of the people.

Government intervention is needed in providing infrastructure for waste collection and appropriate management, waste disposal management through adequate funding, giving preference to recycling wherever possible while existing laws and regulations guiding environmental sanitation and health should be reviewed and enforced with stiffer actions to make them more effective. Solid waste handling, controlling and monitoring techniques in Owerri metropolis must be geared towards achieving quality environmental conditions for man to live in and to protect natural resources such as water that are degraded by these solid wastes.

\section{Declarations}

\section{Source of Funding}

This research did not receive any grant from funding agencies in the public, commercial, or not-for-profit sectors.

\section{Competing Interests Statement}

The authors declare no competing financial, professional and personal interests.

\section{Consent for publication}

Authors declare that they consented for the publication of this research work.

\section{Availability of data and material}

Authors are willing to share data and material according to the relevant needs.

\section{References}

1. Zwane, T.M. (2003) 'Industrial Growth and Water Pollution: Implications and Challenges in Swaziland' [online] (accessed: 14 September, 2008). Available from: http://users.ictp.it/ eee/workshops/smr1597/Zwane.doc.

2. Nnorom, I.C and Osibanjo, O. (2006). Determination of Lead and Cadmium Contents of Dry Cell Batteries Available in Nigeria. J. Appl. Sci. Environ. Mgt., 10(3), 37- 41.

3. Egereonu, U.U, Ukiwe I.N, Edet J.A and Ogukwe C. E. (2012) Investigation of some pollution index of Oramiruikwa, Nworie and Otamiri Rivers, Imo state Nigeria. J. Chem. Soc, Nig. 37(1), 25-29.

4. Ezeronye, O.U. and Ubalua, A.O. (2005). Studies in the effect of abattoir and industrial effluents on the heavy metals and microbial quality of Aba River in Nigeria. Afr. J. Biotechnology. 4(3): 266-272.

5. Fakayode, S.O. (2005). Impact Assessment of Industrial Effluent on Water Quality of the receiving Alaro River in Ibadan, Nigeria. African Journal of Environmental Assessment. Vol.10.

6. Jaiswal, P.C. (2003). Soil, Plant and Water Analysis. Kalyani Pub. New Delhi.

7. Olanrewaju, A.O. (2002). Dangers of indiscriminate refuse dumps in metropolis countries. Environment opinions. 35: 567-571. 
Asian Journal of Basic Science \& Research

Volume 3, Issue 3, Pages 98-109, July-September 2021

8. Onyido, A.E., et al., (2009). A survey of vectors of public health diseases in un-disposed refuse dumps in Akwa town, Anambra state, south eastern Nigeria. Research Journal of Parasitology, 4:22-27.

9. Cookey, P., Kokpan, B.A., Aguo, M.R., Wenes, W. and John, P.C. (2008). Assessment of Water Supply and Sanitation of some Coastal Communities of the Niger Delta. 33rd WEDC International Conference, Accra, Ghana.

10. Nwanebu, F.C (2003). Studies on physico-chemical indicators of pollution in Urashi and Iyiechi Rivers, Imo State, Nigeria. Int. J. Environ. Health Human. Dev, 4(2): 22-28.

11. APHA. (1995). Standard Methods for the Examination of Water and Waste Water. 19th Edition, APHA-AWWA-WPCF. Washington DC. Pp 2.8-4.45.

12. Prescott, I.M, Harley J.P, Klein D.A. (2002). Microbiology. 5th ed. Mc Graw-Hill Inc., NY 10020, 648-688.

13. Ademoroti, C.M.A. (1996), Standard methods for water and effluent analysis, Ibadan Poludex Press, 15-55.

14. Rocchini, R and L. Swain. (2001). 'The British Colombia water quality index Civil and Environmental Research www.iiste.org ISSN 2224-5790 (Paper) ISSN 2225-0514 (Online) Vol.7, No.4, 201553.

15. Osibanjo, O., Daso A.P. and Gbadebo A.M. (2011) The impact of industries on surface water quality of River Ona and River Alaro in Oluyole Industrial Estate, Ibadan, Nigeria Afr. J. Biotechno. 10(4): 696-702.

16. Oboh, I.P. and Edema, C.U. (2007). Levels of heavy metals in water and fishes from the river Niger, J. Chem. Soc. Nig, 32(2) 29-34.

17. Crowther, J., Kay, D., Wyer, M.D. (2001). Relationships between Microbial Water Quality and Environmental Conditions in Coastal Recreational Waters: the Fylde Coast, UK. Water Res. 35:4029 - 4038.

18. Pereira, E, J.A. Baptista-Neto, B.J. Smithand J.J. Mcallister. (2007). The contribution of heavy metal pollution derived from highway runoff to Guanabara Bay sediments--Rio de Janeiro/Brazil. Annals Brazilian Acad. Sci, 79: 739-750.

19. Desai, J. and Tank S.K. (2010). Deterioration of water quality due to immersion of Ganesh idols in the river Tapti at Surat, India, J. Environ. Res. Develop, 4(4), 999-1007.

20. WHO. (2006). Guidelines for drinking water quality, First addendum to the 3rdEd. 1: 491-493.

21. EPTA, 2001, the effect of heavy metals on the environment and on health. http://wwweptanetwork.org/EPTA/ projects.php.

22. Keraita, B, Drechsel, P, Amoah, P. (2003). "Influence of Urban Wastewater on Stream Water Quality and Agriculture in and around Kumasi, Ghana", Environment and Urbanization, 15: 2, 171-178.

23. World Health Organization. (2004). World Health Organization guidelines for drinking water quality (3rd Edition). Geneva, Switzerland. 